\title{
Análise das Interações de Orientadores no Moodle durante a disciplina de Trabalho de Conclusão de Curso a Distância
}

\author{
Cristian Cechinel \\ Faculdade de Educação - Universidade Federal de Pelotas (UFPel) \\ Rua Benjamin Constant, 897 - Pelotas - RS - Brazil \\ contatodcristiancechinel.pro.br
}

\begin{abstract}
The present work describes the analysis of interactions of professors inside a Learning Management System during the Final Undergraduate Project distance discipline. Data about the logs of professors were collected, preprocessed and analyzed in order to observe different aspects of their interactions such as their use of forums and chats and their average number of interactions dedicated per students guided. It was possible to observe that the average number of interactions per student is directly associated to the number of students advised by the professors, and that the two available forms of communication (synchronous and asynchronous) played equally roles during the guidance process.
\end{abstract}

Resumo. O presente trabalho descreve a análise de interações de professores dentro de um Ambiente Virtual de Aprendizagem (AVA) durante uma disciplina de Trabalho de Conclusão de Curso a Distância. Dados sobre os logs de acesso dos professores ao ambiente foram coletados, pré-processados $e$ analisados com o objetivo de observar os diferentes aspectos das suas interações como o uso de fóruns e chats, e o número médio de interações dedicado aos seus orientandos. Foi possível observar que o número médio de interações por estudante está diretamente associado ao número de estudantes orientados pelo professor, e que as duas formas de comunicação disponíveis (síncrona e assíncrona) apresentaram igual importância durante o processo de orientação dos trabalhos.

\section{Introdução}

A Educação a Distância (EAD) no Brasil teve sua expansão marcada pela implantação do programa Universidade Aberta do Brasil (UAB) em 2006. Ao longo desses 8 (oito) anos de existência da UAB, a EAD vem se consolidando como uma das alternativas para a expansão da educação superior em diversas regiões do país, atendendo a aproximadamente 268.028 matrículas (aproximadamente $171.084 \mathrm{em}$ cursos de graduação) espalhadas em mais de 636 polos presenciais. A EAD no Brasil vem também alcançando bons índices de desempenho no ENADE (Exame Nacional de Desempenho de Estudantes), muitas vezes iguais e ou até mesmo superiores aos dos cursos de graduação presenciais (Silva, Oliveira et al. 2012).

As diferentes Universidades que participam da UAB possuem estratégias diferentes na execução de seus projetos, tanto no que se refere ao formato administrativo em que a EAD se estrutura na instituição, como também nos modelos pedagógicos 
adotados em seus cursos. Nessa miríade de possibilidades de trabalho em uma área ainda em consolidação e formação, cada instituição precisa estabelecer também seus parâmetros de qualidade nessa modalidade de educação e conhecer profundamente todos os aspectos que envolvem a execução de suas atividades. Os fomentos fornecidos pelo governo federal, por meio da CAPES para implantar e administrar os cursos a distância dentro da UAB obedecem a um cálculo orçamentário detalhado baseado no número de acadêmicos matriculados em cada turma, assim como nos diversos tipos de atividades acadêmico-administrativas existentes nos cursos como, por exemplo: desenvolvimento de materiais didáticos, supervisão/coordenação de estágios, orientação/coordenação de trabalhos de conclusão de curso, apoio acadêmico, entre outras. Ainda que as instituições possuam esse parâmetro importante para embasar seus planejamentos administrativo e pedagógico, é necessário também buscar novos parâmetros que possam ser contrastados com esses primeiros e que sejam baseados nas experiências obtidas durante a execução das atividades que hoje já acontecem.

O presente trabalho continua um trabalho anterior (Cechinel, Bilhalva et al. 2013) e tem como objetivo iniciar a construção desse corpo teórico baseando-se em dados quantitativos obtidos por meio de um Ambiente Virtual de Aprendizagem (AVA) e durante a execução de uma disciplina de Trabalho de Conclusão de Curso (TCC) do Curso de Licenciatura em Educação do Campo (CLEC) da Universidade Federal de Pelotas (UFPel). Precisamente, o foco do trabalho se restringe a analisar diferentes aspectos das interações dos professores orientadores ao longo do seu trabalho na orientação dos TCCs de seus acadêmicos. É possível encontrar na literatura outros trabalhos também voltados para a análise da execução de disciplinas de TCC à distância. Entretanto, grande parte desses trabalhos apresenta uma metodologia normalmente qualitativa e baseada em questionários de opinião preenchidos por professores e acadêmicos que participaram da disciplina, ou quantitativa, mas voltada para a apresentação das taxas de sucesso e reprovação dos acadêmicos, como por exemplo, em Benfatti, Gomes et al. (2013) e Primo and Silva (2007). Aqui, apresentamos uma perspectiva mais quantitativa e voltada para a interação dos professores orientadores no Moodle e inserida na área que hoje se está denominando de Teaching and Learning Analytics. O objetivo aqui é analisar as interações dos professores orientadores dentro do AVA e descobrir relações que possam auxiliar no planejamento futuro de uma disciplina de TCC desde o ponto de vista administrativo (alocação de professores) como também de organização das salas de aula virtuais.

O restante do trabalho está estruturado da seguinte maneira. A seção 2 apresenta uma breve fundamentação teórica e a seção 3 apresenta a organização da disciplina de TCC do CLEC dentro do AVA (Moodle). A seção 4 descreve a metodologia utilizada no presente trabalho, e a seção 5 analisa e discute os principais resultados encontrados. As conclusões e algumas possibilidades de trabalhos futuros são apresentadas na seção 6.

\section{Fundamentação Teórica}

Já foi estabelecido na literatura de Learning Analytics que o sucesso de estudantes EAD está diretamente correlacionado com o seu engajamento dentro do AVA (AgudoPeregrina, Iglesias-Pradas et al. 2014), (Jayaprakash, Moody et al. 2014), Diferentemente da aprendizagem tradicional, a EAD permite medir o engajamento dos 
estudantes (Beer, Clark et al. 2010) por meio da observação dos logs nos AVAs e da avaliação da intensidade das interações dos estudantes nas diferentes atividades disponíveis nas salas virtuais. A ideia básica é de que quanto mais o estudante interage no AVA, maiores suas chances de sucesso em uma disciplina. A disciplina de TCC é bastante peculiar na medida em que as principais interações dos estudantes são com o seus respectivos orientadores. É claro que as interações entre professores e estudantes são importantes em qualquer tipo de disciplina (Anderson, Liam et al. 2001), entretanto nas disciplinas normais, outros tipos de interações também acontecem em igual proporção e contribuem para o aprendizado e sucesso do estudante (por exemplo, interações com pares, com tutores, e com recursos). No TCC, há primordialmente a interação entre o estudante e seu orientador. Em resumo, "a quantidade e a qualidade das interações do professor está diretamente ligada com a aprendizagem do estudante"(Swan 2003). Como mencionado, a disciplina de TCC é tradada de maneira diferente pela UAB com relação a outras disciplinas, sendo que o orçamento alocado para executá-la depende do número de estudantes que um determinado professor supervisiona. Precisamente, a UAB estabelece que cada professor receberá uma bolsa de dois meses para orientar 5 (cinco) estudantes. Essa é uma referencia que deve ser usada para acomodar as realidades locais de cada instituição dependendo do número de estudantes a ser orientado, e do número de professores disponíveis para essa tarefa. Caso seja possível estabelecer uma associação negativa entre o número de estudantes orientados e o número médio de interações por estudante dos orientadores, isso poderia ajudar os coordenadores dessas disciplinas a planejarem melhor a sua execução. Por exemplo, durante a etapa de planejamento e de contratação dos professores, ao invés de pagar 4 meses de bolsa para um determinado professor orientar 10 (dez) estudantes, seria mais recomendável pagar um professor por menos meses para que o mesmo oriente um número mais reduzido de alunos com o objetivo de não comprometer as interações entre estudantes e orientadores durante a disciplina. O primeiro objetivo desse trabalho pretende avaliar precisamente se existe uma associação entre o número de orientandos alocados para os professores e a quantidade média de suas interações por aluno.

O segundo objetivo do trabalho é avaliar como os professores trabalharam com as ferramentas fornecidas para promover a comunicação com seus orientandos. Como será apresentado na próxima seção, duas formas diferentes de comunicação foram fornecidas: fóruns (assíncrona) e Chats (síncrona). Diferentes trabalhos já foram realizados para avaliar o impacto e a utilidade de cada uma dessas modalidades de comunicação em e-learning. Hrastinski (2008) comparou os benefícios e desvantagens das comunicações assíncrona e síncrona. De acordo com o autor, a comunicação assíncrona é um componente chave para flexibilizar o aprendizado já que permite a comunicação a qualquer momento e em combinação com outras atividades que a pessoa possa estar envolvida (trabalho, família, etc), enquanto que a comunicação síncrona ajuda estudantes e professores a se socializarem melhor e a evitar frustrações a partir da solução de dúvidas em tempo real. Offir, Lev et al. (2008) afirmam que a comunicação assíncrona não promove um diálogo entre estudante e professor e portanto não habilita os estudantes a levantar questões, e que os estudantes preferem aprender por meio da comunicação síncrona. Entretanto, eles ressaltam que estudantes mais habilidosos conseguem superar as interações de baixo nível na comunicação assíncrona. Andresen 
(2009) afirma que a discussão assíncrona em fóruns pode atingir altos níveis de aprendizagem, mas apresenta algumas limitações. $\mathrm{O}$ autor menciona precisamente as dificuldades que os estudantes possuem para se expressarem na modalidade de aprendizagem baseada em problemas, onde as discussões para resolução de problemas são mais específicas e concretas. $\mathrm{O}$ autor menciona que, ao mesmo tempo que os fóruns assíncronos promovem a reflexão, o engajamento e a vontade para discutir em um contexto de educação multicultural, os estudantes sentem que estão envolvidos em uma forma de comunicação com menor significação. Bassett (2011) apresenta um estudo de caso em que estudantes participaram em discussões online assíncronas sobre três artigos de jornais científicos. De acordo com o autor, os estudantes valoraram bem a experiência de aprendizagem e perceberam a comunicação assíncrona como um espaço inclusivo para a colaboração e discussão. Por outro lado, Schultz (2003) descreve um pequeno experimento com seus estudantes em que ele conclui que discussões online síncronas não são tão efetivas quando discussões síncronas presenciais, e sugere que as discussões online síncronas sejam combinadas com outras técnicas de comunicação como os fóruns. Essa pequena revisão da literatura sobre as formas de comunicação em EAD mostra a existência de divergências sobre o assunto e a necessidade de estudos que levem em consideração as realidades locais de cada curso. A análise conduzida nesse trabalho ajudará a avaliar se essas duas modalidades de comunicação estão sendo utilizadas de maneira complementar pelos orientadores no contexto específico da disciplina de TCC a distância.

\section{Organização da disciplina no Moodle}

A disciplina de TCC do CLEC está localizada no último período do curso e possui uma carga horária total de 136 horas/aula executadas em 10 semanas ${ }^{1}$. Para a execução da disciplina de TCC foram criadas duas salas virtuais, sendo elas: Sala de Orientação, e Sala dos Orientadores.

A Sala de Orientação estava destinada a interação entre os orientadores e os acadêmicos, e foi onde o processo de orientação de TCC propriamente dito aconteceu. Nessa sala, as atividades para entrega foram estruturadas em 10 semanas, com atividades definidas para entrega em cada uma das mesmas $\left(1^{\mathrm{a}}\right.$ e $2^{\mathrm{a}}$ semana - Capítulo de Introdução; $3^{\mathrm{a}}$ a $6^{\mathrm{a}}$ semana - Capítulo de Desenvolvimento; $7^{\mathrm{a}}$ e $8^{\mathrm{a}}$ semana - Capítulo de Conclusão; $9^{\mathrm{a}}$ semana - avaliação entrega dos pareceres aos acadêmicos; $10^{\mathrm{a}}$ semana - TCC final). Nessa sala também foram disponibilizados todos os materiais que explicavam o processo pedagógico e gerencial de funcionamento do TCC: ementa, conteúdos, cronograma, atividades, avaliação, referencial teórico, regulamento, normas ABNT, modelo de pareceres de avaliação. Também nessa sala foi disponibilizado um documento geral contendo a equipe de professores orientadores e seus respectivos alunos. A Sala de Orientação foi editada para disponibilizar dois espaços principais para interação entre o professor orientador e o acadêmico: os Fóruns e os Chats. Os Fóruns foram organizados por professor orientador, sendo que de cada Fórum específico de um determinado professor estava organizado internamente com tópicos identificando os nomes de seus acadêmicos orientados (veja exemplo na Figura 1).

Nesses fóruns, cada professor orientava os seus respectivos alunos mediante

\footnotetext{
${ }^{1}$ No período do TCC os acadêmicos cursaram somente essa disciplina e a disciplina de estágio supervisionado.
} 
ser divididos em 4 (quatro) grupos distintos, sendo eles, professores que orientaram: 1 (um) acadêmico, 2 (dois) acadêmicos, 8 (oito) acadêmicos, e 10 (dez) acadêmicos. Essa diferença bem marcada entre o número de orientandos se deve ao fato de que alguns professores orientadores foram selecionados via Edital em que se estabelecia as quantidades de 8 e 10 orientandos para o período de 10 semanas e com dedicação exclusiva a atividade de orientação, enquanto outros professores que já atuavam em outras disciplinas do curso receberam um número mais reduzido de acadêmicos ( 1 ou 2 ) considerando que não dedicariam seu tempo da bolsa ${ }^{2}$ exclusivamente para tal atividade. Cabe ressaltar que além das atividades de orientação, os professores orientadores também atuaram como pareceristas dos TCCs desenvolvidos, sendo que cada professor elaborou uma quantidade de pareceres sobre outros TCCs igual ao dobro do número de TCCs orientados por ele.

\subsection{Pré-Processamento dos Dados}

Um script em Python foi desenvolvido para agrupar os diferentes valores existentes no campo Ação constantes na Tabela 1 de acordo com a atividade principal da mesma. Dessa forma, as diferentes ações executadas em um mesmo tipo de atividade foram agrupadas em somente uma atividade principal, por exemplo, as atividades de visualização da tarefa, de envio da tarefa e de atualização da nota do acadêmico com base na tarefa foram agrupadas em uma única ação denominada Tarefa. As cinco atividades principais resultantes foram: 1) Tarefa, 2) Fórum, 3) Chat, 4) Curso, 5) Recurso, e 6) Usuário.

\section{Análise e Discussão}

O número médio de interações por orientador por acadêmico foi calculado a partir da soma de todas as interações do orientador em determinada sala virtual pelo número de alunos orientados por ele. O número aproximado de interações por professor por aluno variou muito por pessoa em ambas as salas e não apresentou uma distribuição normal em nenhuma delas. Como pode ser visto na Tabela 2, em média, cada orientador realizou aproximadamente 453 interações por aluno na Sala de Orientação (45,3 por aluno por semana)

Tabela 2. Estatísticas Descritivas do número médio de interação por aluno

\begin{tabular}{|c|c|c|c|c|c|c|}
\hline & Média & $\begin{array}{c}\text { Desvio } \\
\text { Padrão }\end{array}$ & $\begin{array}{c}\text { Coeficiente de } \\
\text { Variação }\end{array}$ & $\begin{array}{c}\text { Valor } \\
\text { Mínimo }\end{array}$ & $\begin{array}{c}\text { Valor } \\
\text { Máximo }\end{array}$ & Intervalo \\
\hline $\begin{array}{c}\text { Sala de } \\
\text { Orientação }\end{array}$ & 453,29 & 371,85 & $82,03 \%$ & 93,5 & 1954 & 1860,5 \\
\hline
\end{tabular}

Entretanto o desvio padrão é muito alto nessa sala (371), demonstrando a grande assimetria ocorrida no número médio de interações por aluno por professor. A Figura 2 ilustra essa assimetria.

\footnotetext{
2 O programa UAB oferece bolsas de 20 horas semanais para a atuação de professores no ensino a distância.
} 


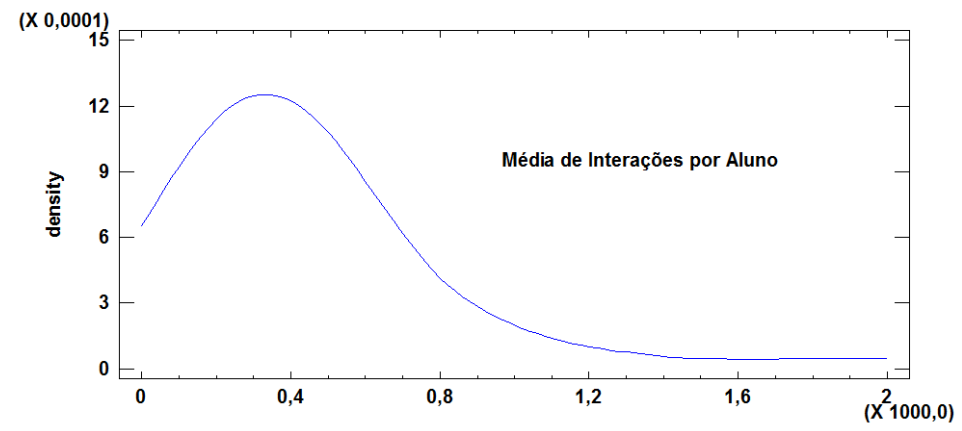

Figura 2. Distribuição de probabilidade da média de interações por aluno dos professores

Como é possível ver na Figura 2, existe uma clara concentração na parte esquerda do gráfico, indicando que a maioria dos orientadores tende a interagir menos (entre o número mínimo e médio de interações) e alguns poucos orientadores tendem a interagir mais (entre o número médio e o número máximo de interações).

\subsection{Interações por categoria de ação}

A Figura 3 apresenta a média de interações dos orientadores por acadêmicos orientados para cada uma das categorias de ações existentes na sala virtual. Como pode ser visto na Figura 3, as duas ações mais frequentes são as relacionadas ao processo de orientação propriamente dito (interação direta entre orientador e acadêmico), ou seja, a utilização dos fóruns como espaços de orientação individualizados (aproximadamente 167 por aluno, representando 37\% do total) e dos chats (134 por aluno, 30\% do total). Ainda, $16 \%$ das interações dos orientadores no ambiente estavam relacionadas à visualização do curso e $13 \%$ ao controle das tarefas dos acadêmicos.

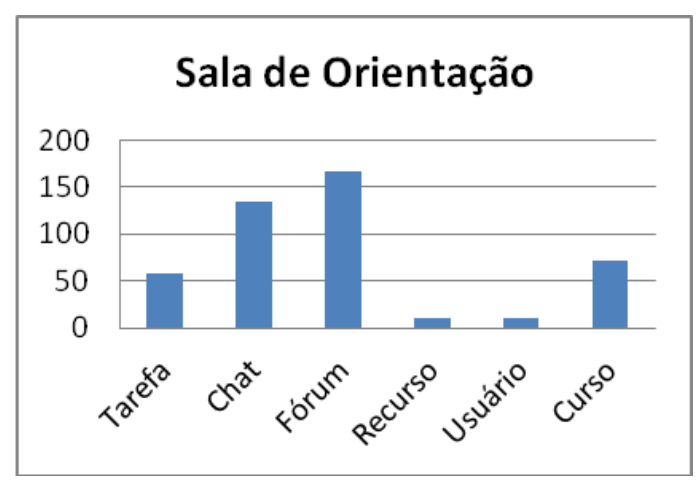

Figura 3. Média de interações dos orientadores por acadêmico por ação

\subsection{Relações com o Número de Orientandos}

Considerando que os professores orientadores que participaram da disciplina de TCC orientaram quantidades diferentes de alunos, foi realizada também uma análise para verificar se essa quantidade tem alguma relação com o número médio de interações por aluno. Tendo em vista que esse número médio não seguia uma distribuição normal (como já mencionado no início dessa seção), um teste de Kruskal-Wallis foi realizado 
para verificar a existência de diferença entre as medianas dos números médios de interações por aluno na Sala de Orientação. $O$ teste apresentou diferença estatisticamente significante entre as medianas do número médio de interações dos professores que orientaram apenas 1 acadêmico para as medianas dos demais grupos (Orientadores de 1 aluno, 2 alunos, 8 alunos e 10 alunos) a um nível de significância de 95\%. A Figura 4 permite melhor a visualização dessas diferenças

Como é possível observar pela Figura 4, existe uma tendência de diminuição do número médio de interações por aluno à medida que o número de alunos orientados pelo professor orientador aumenta. Essa tendência de aumento é mais forte para o grupo de orientadores que possuem 1 (um) orientando.

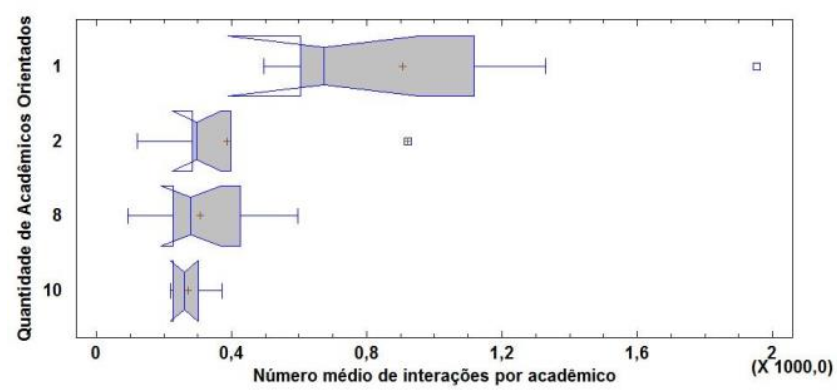

Figura 4. Notched box-plots do número médio de interações por orientando

\subsection{Chat versus Fórum}

Um último experimento foi realizado para avaliar as diferenças nas médias de interações por aluno entre as categorias de ações Chat e Fórum na Sala de Orientação. Um teste de Mann-Whitney (Wilcoxon) foi rodado para verificar se as medianas das duas categorias de ações apresentavam diferenças significativas, e um teste de Kolmogorov-Smirnov foi realizado para verificar se existia diferença entre as distribuições. A Figura 5 apresenta as visualizações dessas análises.

Os testes indicaram que não existe diferença significativa a um nível de confiança de $95 \%$ entre as medianas e as distribuições de ambas as amostras. Isso significa dizer que os trabalhos de orientação síncrono (Chat) e assíncrono (Fórum) podem ser considerados de igual relevância dentro da execução dessa edição da disciplina.
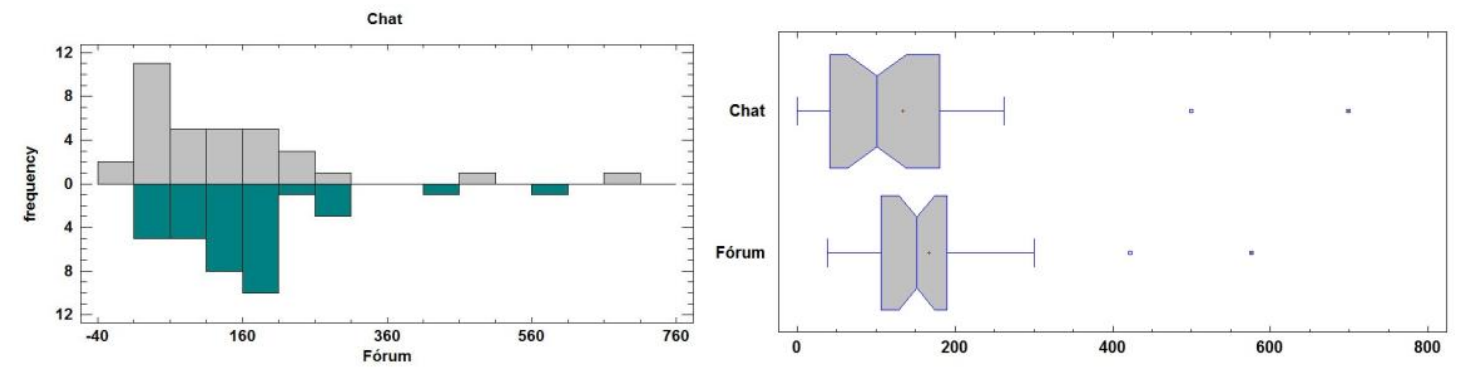

Figura 5. Histogramas (esquerda) e Notched box-plots (direita) para as médias de interações por aluno nas ações Chat versus Fórum 


\section{Conclusões e Trabalhos Futuros}

O presente trabalho apresentou um estudo quantitativo das interações realizadas em duas salas virtuais do Moodle (Sala de Orientação e Sala dos Orientadores) por professores orientadores em uma disciplina de Trabalho de Conclusão de Curso. Para realizar esse trabalho foram coletados registros de log das interações dos professores no ambiente, assim como foi desenvolvido um script em Python para ajudar no préprocessamento das informações coletadas. As análises estatísticas realizadas permitiram descobrir basicamente 2 (dois) principais aspectos sobre a interação dos professores no Moodle.

A primeira descoberta relevante é a relação direta entre o número de acadêmicos orientados e a média de interações dedicadas por aluno por professor. De acordo com a análise realizada, quanto maior o número de acadêmicos orientados, menor é o número médio de interações dedicadas pelo professor aos alunos. É difícil concluir em definitivo sobre as razões pelas quais essa associação negativa existe. Uma possibilidade pode ser o simples fato de que professores que possuem um maior número de orientandos podem ter se sentido sob pressão (com menos tempo para se dedicar a orientação) e consequentemente diminuíram as interações médias com seus alunos. Trabalhos futuros irão focar no estudo das possíveis razões dessa associação assim como também na identificação do número ideal de estudantes que devem ser alocados para cada professor orientador. O segundo aspecto relevante observado por meio das análises é a não existência de diferenças significativamente relevantes entre o número médio de interações por aluno no Chat e no Fórum, ou seja, ambas as ferramentas de orientação foram utilizadas em pé de igualdade pelos orientadores, demonstrando a igual importância que ambas apresentam no processo de orientação do TCC. Análises qualitativas sobre a importância de ambas as formas de comunicação serão realizadas a partir de entrevistas de opinião com alunos e professores para melhor entender as preferências sobre essas modalidades.

Como trabalhos futuros, pretende-se incluir nas análises a quantidade de horas dedicadas por cada professor no processo de orientação e contrastar as mesmas com as ações de interação no ambiente. Para isso, será desenvolvido um segundo script em Python que realizará o cálculo aproximado do tempo de permanência dos professores orientadores dentro do ambiente virtual de aprendizagem Moodle utilizando como base o endereço IP e a data e hora de acesso. É importante lembrar que o trabalho envolvido no processo de orientação do TCC não está restrito ao que acontece dentro do Moodle, uma vez que as leituras e revisões prévias dos trabalhos são realizadas off-line pelos professores. Nesse sentido, o presente trabalho apresenta uma limitação clara, uma vez que ignora em suas análises quaisquer atividades desenvolvidas pelos orientadores fora do ambiente virtual de aprendizagem. Trabalhos futuros podem considerar essas atividades a partir do recolhimento de tais informações diretamente com os professores. Outra importante limitação é a pequena amostra utilizada na análise estatística. Trabalhos futuros irão incorporar mais dados na medida em que novas edições da disciplina forem sendo oferecidas. As descobertas desse estudo auxiliarão no planejamento das próximas edições do Trabalho de Conclusão de Curso e poderão servir como ponto de partida para a criação de parâmetros em EAD baseados nas experiências reais de execução dessa modalidade de educação. 


\section{Referências}

Agudo-Peregrina, Á. F., S. Iglesias-Pradas, M. Á. Conde-González and Á. HernándezGarcía (2014). "Can we predict success from log data in VLEs? Classification of interactions for learning analytics and their relation with performance in VLE-supported F2F and online learning." Computers in Human Behavior 31(0): 542-550.

Anderson, T., R. Liam, D. R. Garrison and W. Archer (2001). "Assessing teacher presence in a computer conferencing context." Journal of the Asynchronous Learning Network 5(2): 17.

Andresen, M. A. (2009). "Asynchronous discussion forums: success factors, outcomes, assessments, and limitations." Journal of Educational Technology \& Society 12(1).

Bassett, P. (2011). "How Do Students View Asynchronous Online Discussions As A Learning Experience?" Interdisciplinary Journal of E-Learning \& Learning Objects 7.

Beer, C., K. Clark and D. Jones (2010). Indicators of engagement. Curriculum, technology \& transformation for an unknown future. Proceedings ascilite Sydney 2010.

Benfatti, E. d. F. S. S., C. Gomes, C. M. d. Costa, L. L. Brasil and S. d. P. Ávila (2013). Orientação de TCC em Ambiente Virtual de Aprendizagem: Uma Pesquisa Ação. $\underline{X}$ Congresso Brasileiro de Ensino Superior a Distância (ESUD 2013). Belém (Pará), UNIREDE.

Cechinel, C., L. Bilhalva and M. Kuskoski (2013). Trabalho de Conclusão de Curso a Distância: Aspectos Quantitativos da Utilização do Ambiente Virtual de Aprendizagem pelos Orientadores. XVIII Congreso Internacional de Informática Educativa (TISE) Nuevas Ideas en Informática Educativa, Porto Alegre.

Hrastinski, S. (2008). "Asynchronous and synchronous e-learning." Educause quarterly 31(4): 51-55.

Jayaprakash, S. M., E. W. Moody, E. J. Lauría, J. R. Regan and J. D. Baron (2014). "Early Alert of Academically At-Risk Students: An Open Source Analytics Initiative." Journal of Learning Analytics 1(1): 6-47.

Offir, B., Y. Lev and R. Bezalel (2008). "Surface and deep learning processes in distance education: Synchronous versus asynchronous systems." Computers \& Education 51(3): 1172-1183.

Primo, L. and C. R. d. O. e. Silva (2007). A Prática de Orientação a Distância na Elaboração de TCCs.

Schultz, R. A. (2003). The effectiveness of online synchronous discussion. Proceedings of the informing science and information technology joint education conference, Finland.

Silva, J. A. R. e., F. B. d. Oliveira and L. Mourão (2012). Uma Comparação entre Cursos a Distância e Presencial. $18^{\circ}$ CIAED Congresso Internacional ABED de EaD "Histórias, Analíticas e Pensamento "Aberto" - Guias para o Futuro da EAD", São Luís (Maranhão).

Swan, K. (2003). "Learning effectiveness online: What the research tells us." Elements of quality online education, practice and direction 4: 13-47. 